RUNNING HEAD: SENSATION-SEEKING, URGENCY, AND RISK-TAKING

\title{
Daily sensation-seeking and urgency in young adults: Examining associations with alcohol
} use and self-defined risky behaviors

McGowan, A.L. ${ }^{1}$, Falk, E.B. ${ }^{1,2,3}$, Zurn, P. ${ }^{4}$, Bassett, D.S..$^{5,6,7,8,9,10}$, \& Lydon-Staley, D.M. ${ }^{1,5,11^{*}}$

${ }^{1}$ Annenberg School for Communication, University of Pennsylvania, Philadelphia, PA

${ }^{2}$ Department of Psychology, University of Pennsylvania, Philadelphia, PA

${ }^{3}$ Marketing Department, Wharton School, University of Pennsylvania, PA

${ }^{4}$ Department of Philosophy and Religion, American University, Washington, DC

${ }^{5}$ Department of Bioengineering, School of Engineering and Applied Science, University of Pennsylvania, Philadelphia, PA, USA

${ }^{6}$ Department of Physics \& Astronomy, College of Arts and Sciences, University of Pennsylvania, Philadelphia, PA, USA

${ }^{7}$ Department of Electrical \& Systems Engineering, School of Engineering and Applied Science, University of Pennsylvania, Philadelphia, PA, USA

${ }^{8}$ Department of Neurology, Perelman School of Medicine, University of Pennsylvania, Philadelphia, PA, USA

${ }^{9}$ Department of Psychiatry, Perelman School of Medicine, University of Pennsylvania, Philadelphia, PA, USA

${ }^{10}$ Santa Fe Institute, Santa Fe, NM, USA

${ }^{11}$ Leonard Davis Institute of Health Economics, University of Pennsylvania, Philadelphia, PA, USA

*Corresponding author: David M. Lydon-Staley, 3620 Walnut St, Annenberg School for Communication, University of Pennsylvania, Philadelphia, PA 19104 USA. Email: david.lydonstaley@asc.upenn.edu 


\section{Citation Diversity Statement}

Recent work in several fields of science has identified a bias in citation practices such that papers from women and other minorities are under-cited relative to the number of such papers in the field [1-9]. Here we sought to proactively consider choosing references that reflect the diversity of the field in thought, form of contribution, gender, race, ethnicity, and other factors. First, we obtained the predicted gender of the first and last author of each reference by using databases that store the probability of a first name being carried by a woman $[6,10]$. By this measure (and excluding self-citations to the first and last authors of our current paper), our references contain $22.22 \%$ woman(first)/woman(last), $13.89 \%$ man/woman, $31.63 \%$ woman/man, and $32.26 \%$ $\mathrm{man} / \mathrm{man}$. This method is limited in that a) names, pronouns, and social media profiles used to construct the databases may not, in every case, be indicative of gender identity and b) it cannot account for intersex, non-binary, or transgender people. We look forward to future work that could help us to better understand how to support equitable practices in science. 


\section{Acknowledgements and Funding Statement}

We would like to thank Asia Vincent, Xinyi Wang, Farah Sayed, Sudeep Bhargava, Anooshey Ikhlas, and Zoe Lebowitz for assistance with thematic analysis of risk reports. D.M.L. and A.L.M. acknowledge support from the National Institute on Drug Abuse (K01 DA047417) and the Brain \& Behavior Research Foundation. D.S.B. acknowledges support from the John D. and Catherine T. MacArthur Foundation, the Alfred P. Sloan Foundation and an NSF CAREER award (PHY-1554488). D.M.L., D.S.B., and P.Z. acknowledge support from the Center for Curiosity. D.M.L., E.B.F., and D.S.B. acknowledge support from the Army Research Office (W911NF-18-1-0244). The views and conclusions contained in this document are those of the authors and should not be interpreted as representing the official policies, either expressed or implied, of the Army Research Office or the U.S. Government. The U.S. Government is authorized to reproduce and distribute reprints for Government purposes notwithstanding any copyright notation herein.

\section{Conflict of Interest Disclosure}

E.B.F. is on the scientific advisory board for kumanu (a digital well being company) and has consulted for google in the past year. The authors have no other conflicts of interest to declare.

\section{Ethics Approval Statement}

All research was conducted in accordance with the Institutional Review Board (IRB) at the University of Pennsylvania.

\section{Permission to Reproduce Material From Other Sources}


Not applicable. 


\begin{abstract}
Purpose: To examine the dynamic expression of sensation-seeking and urgency in daily life and the implications for alcohol use and risk-taking during young adulthood.

Methods: Daily diary surveys were administered to young adults $(n=77)$ aged $18-25$ years every evening for 21 days to assess day's sensation-seeking, urgency, risk-taking, and alcohol use.

Results: Days of higher than usual sensation-seeking are also days of higher than usual risk-taking and are more likely to be alcohol use days than days of lower than usual sensation-seeking. Day's urgency was not associated with day's alcohol use or risk-taking. We extracted 10 themes from self-reports of the day's riskiest behavior: transportation (29.9\%), social (22.8\%), recreation (17.4\%), work (14.8\%), school (13.5\%), food (9.5\%), sleep (9.2\%), substance use $(5.8 \%)$, other $(5.2 \%)$, and jaywalking (1.5\%), and $14.6 \%$ of self-reported risky behaviors were considered threatening to safety, health, or wellbeing.

Conclusions: Risks taken during daily life have mostly positive outcomes and a minority represent threats to safety, health, and wellbeing. Risk-taking and alcohol use in young adult's daily lives is more likely to be driven by the desire to experience novel and exciting experiences than by rash action.
\end{abstract}

Keywords: sensation-seeking; urgency; risk; alcohol; daily diary 


\section{Daily sensation-seeking and urgency in young adults: Examining associations with alcohol use and self-defined risky behaviors}

Young adulthood is characterized by high levels of risk-taking [11,12]. Two traits implicated in risk-taking are sensation-seeking and urgency. Sensation-seeking is the propensity to seek out new, varied, and stimulating experiences, with individuals high in sensation-seeking engaging in risk-taking for the sake of such experiences [13]. Urgency refers to the tendency to act rashly when experiencing intense affect [14], leading to risk-taking resulting from an inadequate consideration of the consequences [15]. In addition to characterizing individuals as high or low in sensation-seeking and urgency, there are within-person fluctuations in sensationseeking and urgency that may impact risk-taking from one day to the next $[16,17]$. We investigate within-person associations among sensation-seeking, urgency, alcohol use, and selfdefined risky behaviors in daily life.

Research using intensive longitudinal methodologies indicate substantial moment-tomoment and day-to-day fluctuations in sensation-seeking and urgency [18]. These fluctuations may have implications for risk-taking. Indeed, initial studies examining within-person associations between day's sensation-seeking (day's urgency was not included in this study) and self-reported risk behavior find that risk-taking was higher than usual on days of higher than usual sensation-seeking, and days of higher than usual sensation-seeking were more likely to be days on which alcohol was consumed relative to days of lower than usual sensation-seeking [17]. These findings suggest that daily fluctuations in the desire for excitement and new experiences are implicated in decisions to drink alcohol and to take risks.

Alcohol is often considered a risky behavior in studies of risk-taking in young adulthood due to the potential negative consequences that may accompany this health risk behavior (e.g., 
dependence, unintentional injury). Yet, risk-taking is not limited to substance use or other illegal or dangerous behaviors that receive substantial treatment in research due to associated public health consequences [19]. Risk-taking is also associated with behaviors that are socially acceptable and constructive, such as adventure, creativity, and exploration [20]. It is especially important to consider risks beyond those with dangerous consequences during the period of young adulthood. This period is characterized by substantial exploration, thus risk-taking may be adaptive, benefit well-being, carry potential costs that are mild in severity, and be socially acceptable [21]. Recent work suggests that focusing on substance use when considering risk behavior fails to capture the majority of behaviors individuals perceive to be risky in their daily lives, illustrating the importance of considering the subjective nature of risk and moving beyond investigator-defined risk constructs [17, 22].

A consideration of a broad array of risk behaviors will also provide insight into how fluctuations in sensation-seeking and urgency motivate risk-taking. There is increasing evidence that trait sensation-seeking is associated with adaptive and exploratory risk-taking carrying positive outcomes whereas impulsigenic traits beyond sensation-seeking, such as urgency, may be more strongly associated with risk-taking associated with negative outcomes [23-25]. There is evidence that day-to-day fluctuations in sensation-seeking are associated with increased risktaking in daily life [17], suggesting that a desire for excitement and new experiences motivates decisions to take risks. However, similar to work on sensation-seeking's daily association with alcohol use, it remains unclear whether the observed associations are specific to sensationseeking. Trait sensation-seeking and trait urgency appear to be differentially related to alcohol use. For example, sensation-seeking has been found to predict drinks per week whereas urgency predicts greater experience of alcohol-related consequences [26]. At the trait level, sensation- 
seeking and other facets of impulsivity such as urgency are often moderately correlated. As such, it is important to control for day's urgency when examining associations among day's sensationseeking, urgency, and risk-taking to ensure that associations are not mistakenly attributed to the included variable (e.g., sensation-seeking) due to the exclusion of the other (e.g., urgency) [27].

\section{The Present Study}

The aims of the present study were to 1) test the extent to which risk-taking in daily life was associated with fluctuations in sensation-seeking and/or urgency and 2) gain insight into the types, and outcomes, of risks that are undertaken during daily life. We include alcohol as a canonical risk behavior $[16,17]$ and additionally collect information on self-reported risks. In line with previous work [17], we hypothesized that days of higher than usual sensation-seeking would be days of higher than usual risk-taking and days on which alcohol use is consumed. No association between day's sensation-seeking and amount of alcohol consumed was hypothesized, in line with previous work at the trait and daily level [17, see 28 for a review]. Building on findings at the trait level $[18,28]$, we hypothesized that sensation-seeking's associations with alcohol use and risk-taking would be independent from day's urgency. In line with previous findings [17], we hypothesized that a small proportion of self-reported risks would involve substance use and be considered threatening to safety, health, or wellbeing. As such, we hypothesized no significant associations between day's urgency and day's risk-taking and alcohol use since urgency tends to be associated with more extreme risk behaviors, such as alcohol problems and risks with negative consequences $[29,30]$, than have been previously observed at the daily level [17].

\section{Method}


All research was conducted in accordance with the Institutional Review Board (IRB) at the University of Pennsylvania.

\section{Participants}

A final sample of 77 young adults $(M=21.2$ years, $S D=1.75,63$ women, see Table 1 for additional participant demographic characteristics) participated in this study. Participants were recruited from the University of Pennsylvania and the surrounding university community through poster, Facebook, Craigslist, and university research site advertisements. Individuals were eligible if they met 5 criteria: 1) were between the ages of 18 and 25 years of age; 2) had consistent home access to a desktop or laptop with internet; 3) owned a smartphone; 4) were willing to complete a 2-hour laboratory visit; and 5) were willing to install a free app on their smartphone and computer. An initial sample of 80 participants was recruited; however, 3 participants were excluded for non-compliance with the daily diary and smartphone-based experience sampling protocol (i.e., having no instances of the daily diary or experience-sampling data).

\section{Procedure}

Participants attended a laboratory visit and then completed 21 days of daily diaries and an experience-sampling assessment. We focus on data from the laboratory visit and the daily diary in the present manuscript. Links to the end-of-day daily diary surveys were sent via email at 6:30pm every evening. Participants were compensated with a payment card that could be used as a debit card (see supplemental materials for payment details). Data collection began in July 2019 and ended in March 2020 when laboratory visits were no longer possible due to COVID-19.

\section{Measures}


We used participants' reports of demographic characteristics from the laboratory session and their daily diary reports.

Day's Sensation-Seeking. Day's sensation-seeking was measured using two items adapted from the Fun-Seeking subscale of the BIS/BAS scales [31] and the Excitement-Seeking subscale of the Revised Neuroticism, Extraversion, and Openness Personality Inventory [32]. To capture day's sensation-seeking, participants responded to the following two questions, the average of which was then taken to create a day's sensation-seeking scale: “Today, I craved excitement" and "Today, I craved new experiences". The sensation-seeking scale exhibited reliable within-person change $\left(R_{c}=0.87\right)$. Intraclass correlation analyses indicated that the proportion of variance associated with between-person variability in day's sensation-seeking was 0.66. The mean of the day's sensation-seeking score showed positive and moderate correlations with two trait measures of sensation-seeking, the Brief Sensation Seeking Scale $(r=0.34, p=$ $0.003)$ and the UPPS sensation-seeking subscale $(r=0.35, p=0.002)$, providing evidence for the scale's convergent validity. Correlations between the day's sensation-seeking scale and the premeditation, urgency, and perseverance UPPS subscales were not significant $(r$ 's $\leq-0.10, p$ ' $s \geq$ 0.28), providing evidence for discriminant validity of the scale.

Day's Urgency. Day's urgency was measured using two items adapted for daily use from the urgency subscale of the Urgency, Premeditation, Perseverance, and Sensation-Seeking Scale (UPPS) [14]. To capture day's urgency, participants responded to the following two questions, the average of which was then taken to create a day's urgency scale: “Today, I had trouble keeping my feelings under control”, “Today, I had trouble controlling my impulses”. Participants provided their responses to these daily items on a sliding scale from 1 ("not at all") to 100 ("very") in increments of 1 . The urgency scale exhibited reliable within-person change $\left(R_{c}=\right.$ 
0.79). Intraclass correlation analyses indicated that the proportion of variance associated with between-person variability in day's urgency was 0.52 . The mean of the day's urgency score showed positive and moderate correlations with one trait measure of urgency, the UPPS urgency subscale $(r=0.47, p<0.001)$, providing evidence for the scale's convergent validity. Day's urgency was not significantly correlated with the Brief Sensation Seeking Scale $(r=0.09, p=$ 0.44) and correlations between the day's urgency scale and the premeditation, sensation-seeking, and perseverance UPPS subscales were not significant ( $r$ 's $\leq-0.16, p$ 's $\geq 0.11$ ) providing evidence for discriminant validity of the scale. Day's sensation-seeking and day's urgency were not significantly correlated $\left(r_{r m}=0.05, p=0.68\right)$.

Day's Risk-Taking. Day's risk-taking was measured during the morning survey using the mean of two items used in a previous study on daily risk-taking [17]: "Yesterday, I took more or less risks than I normally do" and "Yesterday, my behavior was more or less risky than usual" on a slider with the anchors: -50 (less than usual), 0 (same as usual), and 50 (more than usual). Participants were asked to report on yesterday's riskiest behavior to ensure risky behaviors that may have occurred following completion of the evening survey were captured. The mean of all day's risk-taking reports in the dataset was $-1.2(S D=17.73, \min =-50, \max =50)$. The risktaking scale exhibited reliable within-person change $\left(R_{c}=0.93\right)$. Intraclass correlation analyses indicated that the proportion of variance associated with between-person variability in day's risktaking was 0.24 .

To provide insight into the types of risks participants engaged in during daily life, participants also reported on their riskiest behavior of the day in an open-ended response to the question in the morning survey based on the previous day: "What was the riskiest thing you did yesterday?". A content analysis of the self-reported risky behaviors indicated highly 
idiosyncratic risks. Yet, 10 common themes emerged (see Figure S1 for more detail):

transportation (29.9\%), social (22.8\%), recreation (17.4\%), work (14.8\%), school (13.5\%), food (9.5\%), sleep (9.2\%), substance use (5.8\%), other (5.2\%), and jaywalking (1.5\%). The average Fleiss' kappa [33] was 0.77 and average pairwise agreement among the three coders was $94.4 \%$, indicating substantial agreement among coders.

To complement the thematic analysis of riskiest behaviors of the day, three researchers independently coded each of the 1147 reported risks as either "not threatening to safety, health, or wellbeing" or "threatening to safety, health, or wellbeing" (see [17] for a similar treatment). Of the 1147 reported risks, $167(14.6 \%)$ were unanimously coded as risks that are threatening to safety, health, or wellbeing. Example risks coded as threatening to safety, health, or wellbeing include: "crossed the street while cars were coming", "take the bus home alone late at night", "stand on platform to see fireworks better", "getting drunk alone". The percent of risk behaviors unanimously coded as threatening within each category is shown in Table S1. Jaywalking (100\%), substance use (90.2\%), and transportation (34.6\%) risk categories were among the risk categories with the greatest percent of risks threatening to safety, health, or wellbeing. We report the percent of reports unanimously coded within each category in Table S2.

Participants also rated the outcome of the riskiest behavior according to two separate items using a scale from 1 ("Not at all") to 100 ("Very") in increments of 1: "The outcome of the riskiest thing you did yesterday was positive" and "The outcome of the riskiest thing you did yesterday was negative".

Day's Alcohol Use. Alcohol consumption for the previous day was measured during each daily diary assessment using three items of the form, "Yesterday, how many of the following drinks did you consume?" followed with prompts and definitions of standard servings 
for beer (12 fl. oz.), wine (5 fl. oz.), and shots of liquor (1.5 fl. oz.). Responses were given on a $0,1,2,3,4,5+$ response scale for each beverage category and were summed to obtain the total servings of alcohol consumed the previous day. Participants were asked to report on yesterday's alcohol use to ensure that alcohol use that may have occurred after completion of the evening survey was captured.

\section{Statistical Analysis}

Data preparation and procedures common to models for day's alcohol and day's risktaking are described here before procedures specific to the alcohol and risk-taking variables are described. We parameterized the time-varying daily diary variables (day's sensation-seeking and day's urgency) to separate within-person and between-person associations by splitting predictors into time-invariant (between-person; which we refer to as usual sensation-seeking) and timevarying (within-person; which we refer to as day's sensation-seeking) components [34]. We slid forward the risk-taking and alcohol use variables by one day (as the questions were phrased to measure yesterday's risk-taking and alcohol use). This ensured that today's sensation-seeking and urgency predicted today's risk-taking and alcohol. Social weekend was used to code for weekend days such as Thursday, Friday, and Saturday, which are considered days when alcohol use is most likely in young adults and college students [35]. We included usual sensationseeking, usual urgency, weekend (dummy coded such that Thursday, Friday, and Saturday were indicated by 1$)$, day of study (to account for time as a third variable), age, and gender $(0=$ woman; $1=$ man) as covariates in all models. We specified random intercepts and random slopes for day of study, day's sensation-seeking, and day's urgency in all models. The model for risktaking did not converge when a random effect for day's urgency was included and, as such, a 
more parsimonious model without this random effect estimated is presented. All analyses were performed using R Studio version 1.1.463 [36].

Associations among daily sensation-seeking, urgency, and risk-taking. We tested the association between day's sensation-seeking and day's urgency (predictors) with day's risktaking (outcome) using multilevel model to account for the nesting of the data (days nested in participants) using the nlme package in $\mathrm{R}$ [37]. In an exploratory analysis, we used a multilevel logistic model to test the extent to which day's sensation-seeking and day's urgency predicted whether the riskiest behavior of the day was coded as a risk that was "not threatening to safety, health, or wellbeing" (0) or "threatening to safety, health, or wellbeing" (1).

\section{Associations between daily sensation-seeking, urgency, and risk-taking and alcohol}

use. We next tested the extent to which day's sensation-seeking and urgency (predictors) were associated with day's alcohol use (outcome) by fitting a multilevel hurdle model using glmmTMB package [38], specifying a truncated negative binomial function. Count data, such as the day's alcohol use variable reported on in this manuscript (i.e., number of drinks consumed), are often positively skewed and include many observations at zero. These data violate the assumption of linear mixed models. Hurdle models include a logistic regression to model the zeroes in the data in addition to a count regression (in this case negative binomial) to model the counts. All the zeroes (nonalcohol use days) are modeled with the logistic regression and nonzero-counts (alcohol use days) are modeled by a negative binomial function. The zeroinflation submodel of the hurdle model estimates the probability of an extra zero (no alcohol use) such that a positive estimate indicates a higher chance of no alcohol use. The conditional submodel of the hurdle model estimates the positive count process, providing insight into 
variables that increase or decrease the likelihood of consuming more alcohol on alcohol use days.

\section{Results}

Out of a possible total of 1617 daily diary days (21 days $\times 77$ participants), 1119 (69.2 \%) were available. The number of daily diary days completed by participants ranged from 1 to $20(M=14.53, S D=5.02)$. The number of study days completed was unrelated to day's sensation-seeking or day's urgency ( $r$ 's $\leq-0.21$ [95\% CI: -0.42 to 0.18 ], $p$ 's $\geq 0.08$ ). We provide descriptive statistics and correlations of the variables used in the analyses in Table S3.

\section{Day's sensation-seeking, urgency, and alcohol use}

Days of higher than usual sensation-seeking were more likely to be days on which alcohol was used $(b=-0.01, p=0.04$; see Table 2; Figure 1A). There was no association between day's urgency and alcohol use $(b=0.003, p=0.59)$. Neither day's sensation-seeking nor day's urgency were associated with amount of alcohol consumed ( $b{ }^{\prime} s \leq 0.002, p{ }^{\prime} s \geq 0.53$ ).

\section{Day's sensation-seeking, urgency, and risk-taking}

Days of higher than usual sensation-seeking were also days of higher than usual risktaking $(b=0.10, p=0.008$; see Table 3, Figure 1B). Day's urgency was unrelated to risk-taking $(b=0.02, p=0.56)$. To provide greater context to the finding that participants reported riskier behavior on days of higher than usual sensation-seeking, we examined the self-reported outcome of these risky behaviors. The outcomes of risks were rated as generally high in positive outcomes $(M=70.11, S D=26.37)$ and low on negative outcomes $(M=23.2, S D=24.56)$ on average as calculated using the sample-mean of the intraindividual mean. Additional exploratory analyses testing the extent to which day's sensation-seeking and day's urgency predicted whether the selfreported riskiest behavior of the day was coded as a risk that was "not threatening to safety, 
health, or wellbeing" or "threatening to safety, health, or wellbeing" revealed no significant associations with day's sensation-seeking or day's urgency.

\section{Discussion}

We measured naturalistic daily fluctuations in sensation-seeking and urgency over the course of 21 days and tested within-person associations between day's sensation-seeking, urgency, and both self-defined risky behavior and alcohol use. We find that days of higher than usual sensation-seeking were also days of higher than usual risk-taking and were more likely to be alcohol use days relative to low sensation-seeking days. Notably, sensation-seeking's withinperson association with alcohol was specific to normative engagement with alcohol use during young adulthood rather than heavy alcohol consumption that poses a more serious threat to wellbeing [39]. These findings replicate prior work [17] and extend it by examining the distinct within-person associations between sensation-seeking and both self-reported risk-taking and alcohol use independent from urgency. Findings suggest that increases in risk-taking in the daily lives of young adults are more likely to be driven by increases in the desire to experience novel and stimulating sensations than increases in rash action.

We additionally provide insight into the types and outcomes of risks undertaken in daily life by young adults. Risks had positive outcomes on average with few reports of negative outcomes. Content analysis revealed highly idiosyncratic risks, with common themes emerging consistent with prior work in this area [17]. These insights into daily risks further support the hypothesized distinct roles of sensation-seeking and urgency in risk-taking [21,24,25]. Increases in state sensation-seeking during daily life, while predictive of increases in risk-taking, seem seldomly accompanied by negative risk behaviors that are dangerous and imperil wellbeing. The lack of significant associations between day's urgency and risk behaviors likely stems in part from the 
nature of risks undertaken in participants' daily lives. Urgency has been shown to be associated with maladapative risk-taking [40,41] and only a minority of the self-reported risky behaviors posed a threat to safety, health, and wellbeing.

\section{Limitations and Future Outlook}

The sample is primarily high functioning, white, college-aged adults identifying as women with no alcohol-related impairment or pathology, making generalization to other samples difficult. The self-report measures of alcohol use and risk-taking were retrospective in nature (reporting on the previous day's behaviors). This approach allowed us to capture behaviors occurring after the daily survey was sent $(6: 30 \mathrm{pm})$ but may have introduced retrospective biases. The focus on daily associations between sensation-seeking and alcohol use and risky behaviors allowed us to replicate previous work [17] and extend it by including a measure of daily urgency. Although the daily associations between sensation-seeking, alcohol use, and risk-taking were replicated, experience-sampling work capturing within-day measures of sensation-seeking and alcohol have observed different associations [16]. In particular, momentary sensation-seeking was associated with amount of drinking, an association we did not observed at the day-level. In addition to timescale differences between these two studies, there are differences in the operationalization of sensation-seeking. The present manuscript used a two-item sensationseeking scale tapping the desire for new and exciting experiences, which showed strong discriminant validity from day's urgency ( $r=0.05$; Table S3). The one-item momentary sensation-seeking scale in Griffin and Trull [16] assessed engagement in behaviors motivated by thrill-seeking ("I did something for the thrill of it") that showed strong correlations with momentary urgency $(r=0.83)$ which may partly explain the few associations observed between sensation-seeking and alcohol use in that study. Continued considerations of how best to measure 
sensation-seeking and other impulsigenic states on shorter timescales relative to more traditional, trait assessments will be key.

\section{Conclusion}

In conclusion, the risks young adults take during daily life have positive outcomes on average with few reports of negative outcomes. Day's risk-taking and alcohol use is significantly associated with day's sensation-seeking but not day's urgency, suggesting that risk-taking in the daily lives of young adults without alcohol-related impairment or pathology is likely to be driven more by the desire to experience novel and stimulating sensations than by rash action. 


\section{References}

[1] Bertolero MA, Dworkin JD, David SU, et al. Racial and ethnic imbalance in neuroscience reference lists and intersections with gender. BioRxiv 2020.

[2] Caplar N, Tacchella S, Birrer S. Quantitative evaluation of gender bias in astronomical publications from citation counts. Nat Astron 2017;1:1-5.

[3] Chakravartty P, Kuo R, Grubbs V, et al. \# CommunicationSoWhite. J Commun 2018;68:254-66.

[4] Dion ML, Sumner JL, Mitchell SM. Gendered citation patterns across political science and social science methodology fields. Polit Anal 2018;26:312-27.

[5] Wang X, Dworkin J, Zhou D, et al. Gendered Citation Practices in the Field of Communication 2020.

[6] Dworkin JD, Linn KA, Teich EG, et al. The extent and drivers of gender imbalance in neuroscience reference lists. ArXiv Prepr ArXiv200101002 2020.

[7] Fulvio JM, Akinnola I, Postle BR. Gender (im) balance in citation practices in cognitive neuroscience. J Cogn Neurosci 2020:1-5.

[8] Maliniak D, Powers R, Walter BF. The gender citation gap in international relations. Int Organ 2013;67:889-922.

[9] Mitchell SM, Lange S, Brus H. Gendered citation patterns in international relations journals. Int Stud Perspect 2013;14:485-92.

[10] Zhou D, Cornblath EJ, Stiso J, et al. Gender diversity statement and code notebook v1. 0. 2020. URL Httpsdoi Org105281zenodo 2020;3672110.

[11] Krieger H, Young CM, Anthenien AM, et al. The epidemiology of binge drinking among college-age individuals in the United States. Alcohol Res Curr Rev 2018;39:23-30.

[12] Li K, Simons-Morton B, Gee B, et al. Marijuana-, alcohol-, and drug-impaired driving among emerging adults: Changes from high school to one-year post-high school. J Safety Res 2016;58:15-20.

[13] Zuckerman M. Behavioral Expressions and Biosocial Bases of Sensation Seeking. Cambridge University Press; 1994.

[14] Whiteside SP, Lynam DR. The five factor model and impulsivity: Using a structural model of personality to understand impulsivity. Personal Individ Differ 2001;30:669-89.

[15] Cyders MA, Flory K, Rainer S, et al. The role of personality dispositions to risky behavior in predicting first-year college drinking. Addiction 2009;104:193-202.

[16] Griffin SA, Trull TJ. Alcohol use in daily life: Examining the role of trait and state impulsivity facets 2020 .

[17] Lydon-Staley DM, Falk EB, Bassett DS. Within-person variability in sensation-seeking during daily life: Positive associations with alcohol use and self-defined risky behaviors. Psychol Addict Behav 2020;34:257-68.

[18] Halvorson MA, King K, Feil M. Impulsive states and impulsive traits: A study of the multilevel structure and validity of a multifaceted measure of impulsive states 2019.

[19] Kann L, McManus T, Harris WA, et al. Youth Risk Behavior Surveillance — United States, 2017. MMWR Surveill Summ 2018;67:1-114.

[20] Hansen EB, Breivik G. Sensation seeking as a predictor of positive and negative risk behaviour among adolescents. Personal Individ Differ 2001;30:627-40.

[21] Duell N, Steinberg L. Positive Risk Taking in Adolescence. Child Dev Perspect 2019;13:48-52.

[22] Hansson SO. Risk: objective or subjective, facts or values. J Risk Res 2010;13:231-8. 
[23] Khurana A, Romer D, Betancourt LM, et al. Modeling Trajectories of Sensation Seeking and Impulsivity Dimensions from Early to Late Adolescence: Universal Trends or Distinct Sub-groups? J Youth Adolesc 2018;47:1992-2005.

[24] Romer D, Reyna VF, Satterthwaite TD. Beyond stereotypes of adolescent risk taking: Placing the adolescent brain in developmental context. Dev Cogn Neurosci 2017;27:19-34.

[25] Yoneda T, Ames ME, Leadbeater BJ. Is there a positive side to sensation seeking? Trajectories of sensation seeking and impulsivity may have unique outcomes in young adulthood. J Adolesc 2019;73:42-52.

[26] LaBrie JW, Kenney SR, Napper LE, et al. Impulsivity and alcohol-related risk among college students: Examining urgency, sensation seeking and the moderating influence of beliefs about alcohol's role in the college experience. Addict Behav 2014;39:159-64.

[27] Magid V, MacLean MG, Colder CR. Differentiating between sensation seeking and impulsivity through their mediated relations with alcohol use and problems. Addict Behav 2007;32:2046-61.

[28] Coskunpinar A, Dir AL, Cyders MA. Multidimensionality in Impulsivity and Alcohol Use: A Meta-Analysis Using the UPPS Model of Impulsivity. Alcohol Clin Exp Res 2013;37:1441-50.

[29] Mundt MP, Zakletskaia LI, Fleming MF. Extreme College Drinking and Alcohol-Related Injury Risk. Alcohol Clin Exp Res 2009;33:1532-8.

[30] Cherpitel CJ. Alcohol, injury, and risk-taking behavior: data from a national sample. Alcohol Clin Exp Res 1993;17:762-6.

[31] Carver CS, White TL. Behavioral inhibition, behavioral activation, and affective responses to impending reward and punishment: the BIS/BAS scales. J Pers Soc Psychol 1994;67:319.

[32] Costa PT, McCrae RR. Manual for the revised NEO personality inventory (NEO-PI-R) and NEO five-factor inventory (NEO-FFI). Odessa FL Psychol Assess Resour 1992.

[33] Fleiss JL. Measuring nominal scale agreement among many raters. Psychol Bull 1971;76:378.

[34] Bolger N, Laurenceau J-P. Intensive longitudinal methods: An introduction to diary and experience sampling research. Guilford Press; 2013.

[35] Finlay AK, Ram N, Maggs JL, et al. Leisure Activities, the Social Weekend, and Alcohol Use: Evidence From a Daily Study of First-Year College Students. J Stud Alcohol Drugs 2012;73:250-9.

[36] R Core Team. R: A language and environment for statistical computing. Vienna, Austria: 2019.

[37] Pinheiro J. nlme: linear and nonlinear mixed effects models. R package version 3.1-96. Httpcran R-Proj Orgwebpackagesnlme 2009.

[38] Brooks ME, Kristensen K, van Benthem KJ, et al. glmmTMB balances speed and flexibility among packages for zero-inflated generalized linear mixed modeling. R J 2017;9:378-400.

[39] NSDUH. National Survey on Drug Use and Health (NSDUH-2018). Available at: https://www.datafiles.samhsa.gov/study/national-survey-drug-use-and-health-nsduh-2018nid18757. AccessedOctober 30, 2020.

[40] Cyders MA, Smith GT. Emotion-based dispositions to rash action: positive and negative urgency. Psychol Bull 2008;134:807. 
[41] Cyders MA, Dzemidzic M, Eiler WJ, et al. Negative Urgency Mediates the Relationship between Amygdala and Orbitofrontal Cortex Activation to Negative Emotional Stimuli and General Risk-Taking. Cereb Cortex 2015;25:4094-102. 
Table 1. Participant demographic characteristics

\begin{tabular}{|c|c|}
\hline Measure & All Participants \\
\hline $\mathrm{N}$ & 77 (63 female) \\
\hline Age (years) & $21.2 \pm 1.8$ \\
\hline \multirow[t]{6}{*}{ Race/Ethnicity } & African American/Black (20.8\%) \\
\hline & Asian-American (23.4\%) \\
\hline & Hispanic/Latino (9.1\%) \\
\hline & Multiracial (7.8\%) \\
\hline & Other $(7.8 \%)$ \\
\hline & white $(31.2 \%)$ \\
\hline \multirow[t]{5}{*}{ Education } & less than a high school degree $(1.3 \%)$ \\
\hline & high school degree $(28.2 \%)$ \\
\hline & some college with no degree $(42.3 \%)$ \\
\hline & bachelor's degree (26.9\%) \\
\hline & master's degree $(1.3 \%)$. \\
\hline \multirow[t]{5}{*}{ Employment } & full-time student (47.9\%) \\
\hline & part-time student $(24.7 \%)$ \\
\hline & part-time worker $(6.5 \%)$ \\
\hline & full-time worker $(9.1 \%)$ \\
\hline & unemployed (11.7\%) \\
\hline
\end{tabular}


Table 3. Results of the multilevel models examining associations of day's sensation-seeking and urgency with day's risk-taking.

\begin{tabular}{|c|c|c|c|c|c|c|}
\hline \multirow[b]{2}{*}{ Effect } & \multirow[b]{2}{*}{ Estimate } & \multirow[b]{2}{*}{$\begin{array}{l}\text { Standard } \\
\text { error }\end{array}$} & \multirow[b]{2}{*}{$p$} & \multirow[b]{2}{*}{$d$} & \multicolumn{2}{|c|}{ Confidence interval } \\
\hline & & & & & Lower & Upper \\
\hline \multicolumn{7}{|l|}{ Fixed effects } \\
\hline Intercept & -2.16 & 1.26 & 0.09 & & -4.63 & 0.31 \\
\hline Day of study & -0.13 & 0.12 & 0.26 & -0.07 & -0.37 & 0.10 \\
\hline Gender man & $-6.22 *$ & 2.86 & 0.03 & -0.51 & -11.92 & -0.52 \\
\hline Weekend & $3.05 * *$ & 0.95 & 0.001 & 0.20 & 1.18 & 4.91 \\
\hline Age & 0.10 & 0.64 & 0.87 & 0.04 & -1.16 & 1.37 \\
\hline Day's sensation-seeking & $0.10^{*}$ & 0.04 & 0.008 & 0.17 & 0.03 & 0.17 \\
\hline Usual sensation-seeking & $0.16^{* *}$ & 0.05 & 0.001 & 0.83 & 0.07 & 0.25 \\
\hline Day's urgency & 0.02 & 0.03 & 0.56 & 0.04 & -0.04 & 0.08 \\
\hline Usual urgency & 0.01 & 0.06 & 0.88 & 0.04 & -0.11 & 0.13 \\
\hline Random effects & \multicolumn{2}{|c|}{ Variance } & & & \multicolumn{2}{|c|}{ Standard Deviation } \\
\hline Intercept & \multicolumn{2}{|c|}{68.80} & & & \multirow{2}{*}{\multicolumn{2}{|c|}{$\begin{array}{l}8.23 \\
0.8\end{array}$}} \\
\hline Day of study & \multicolumn{2}{|c|}{0.47} & & & & \\
\hline Day's sensation-seeking & \multicolumn{2}{|c|}{0.02} & & & \multirow{2}{*}{\multicolumn{2}{|c|}{0.13}} \\
\hline Residual & \multicolumn{2}{|c|}{211.45} & & & & 14.54 \\
\hline
\end{tabular}

Note. 1095 observations nested within 77 participants. $* p<0.05, * * p \leq 0.001$. 
Table 2. Results of multilevel hurdle model examining associations among sensation-seeking, urgency, and alcohol use.

\begin{tabular}{lccc}
\hline \multicolumn{1}{c}{ Effect } & Estimate & $\begin{array}{c}\text { Standard } \\
\text { error }\end{array}$ & $p$ \\
\hline & \multicolumn{3}{c}{ Conditional submodel } \\
Fixed effects & $0.51^{*}$ & 0.14 & 0.0004 \\
Intercept & 0.02 & 0.01 & 0.07 \\
Day of study & $0.31^{*}$ & 0.13 & 0.02 \\
Weekend & -0.01 & 0.07 & 0.85 \\
Age & 0.10 & 0.30 & 0.97 \\
Gender man & 0.002 & 0.004 & 0.54 \\
Day's sensation-seeking & -0.001 & 0.005 & 0.91 \\
Usual sensation-seeking & -0.002 & 0.004 & 0.60 \\
Day's urgency & 0.004 & 0.005 & 0.47 \\
Usual urgency & Variance & SD \\
\hline Random effects & 0.10 & 0.31 \\
Intercept & $<0.001$ & $<0.001$ \\
Day of study & 0.07 & 0.26 \\
Day's sensation-seeking & \multicolumn{2}{c}{0.05} & 0.23 \\
Day's urgency & \multicolumn{1}{c}{ Effect } & Estimate & Standard \\
& \multicolumn{3}{c}{ error } \\
\hline
\end{tabular}

Fixed effects

\begin{tabular}{lccc} 
Intercept & $2.57^{* *}$ & 0.29 & $<0.001$ \\
Day of study & 0.002 & 0.02 & 0.92 \\
Weekend & $-0.86^{* *}$ & 0.21 & $<0.001$ \\
Age & $-0.22^{*}$ & 0.11 & 0.04 \\
Gender man & 0.48 & 0.48 & 0.32 \\
Day's sensation-seeking & $-0.01^{*}$ & 0.006 & 0.04 \\
Usual sensation-seeking & -0.01 & 0.008 & 0.07 \\
Day's urgency & 0.003 & 0.006 & 0.59 \\
Usual urgency & $-0.02^{*}$ & 0.10 & 0.03 \\
\hline Random effects & Variance & & $\mathrm{SD}$ \\
\hline Intercept & 1.65 & & 1.28 \\
Day of study & 0.07 & & 0.27 \\
Day's sensation-seeking & $<0.001$ & & 0.001 \\
Day's urgency & $<0.001$ & & 0.001 \\
\hline
\end{tabular}

Note. 1097 observations nested within 77 participants. The zero-inflation submodel of the hurdle model estimates the probability of an extra zero (no alcohol use) such that a positive estimate indicates a higher chance of no alcohol use. $* p<0.05, * * p<0.001$ 


\section{Figure Captions}

Figure 1. Graphic representation of (A) the multilevel hurdle model showing the association of day's sensation-seeking with alcohol use and (B) the multilevel model showing the association of day's sensation-seeking with day's risk-taking. $95 \%$ confidence intervals are represented in gray. 
Figure 1.

A)

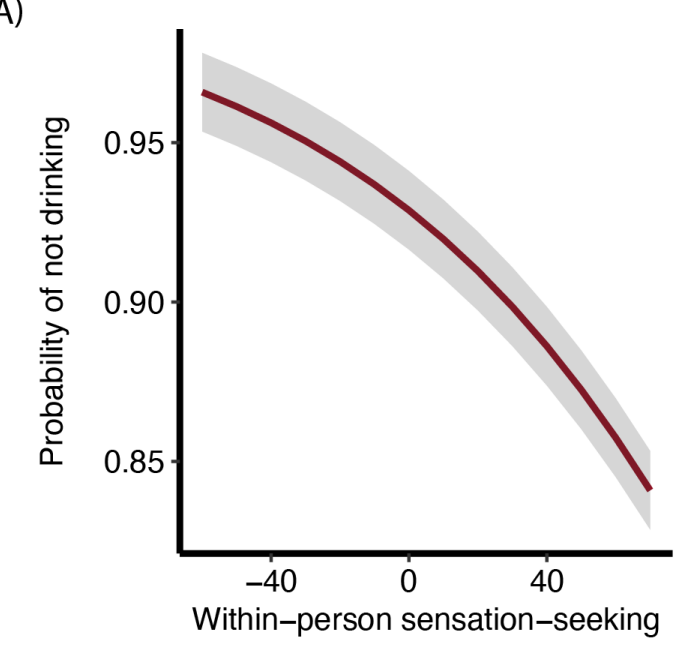

B)

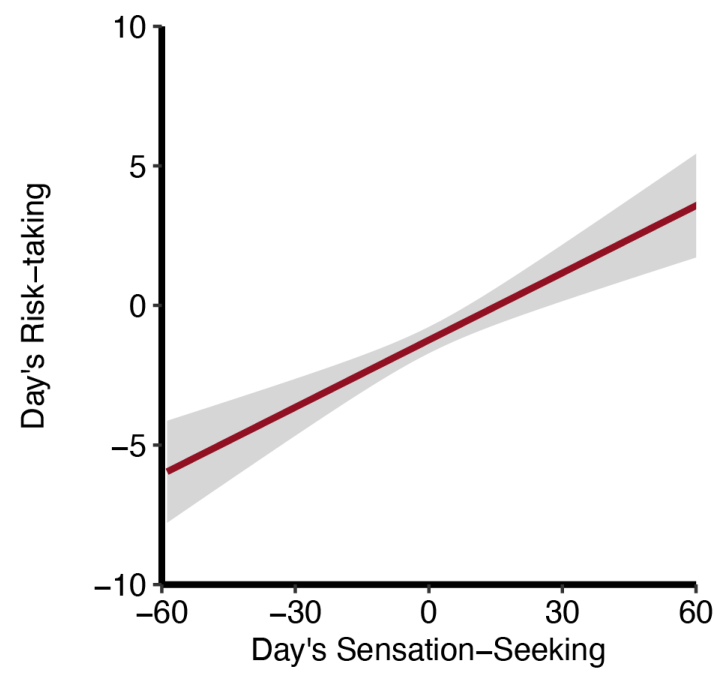

